\title{
LncRNA TTN-AS1 contributes to gastric cancer progression by acting as a competing endogenous RNA of miR-376b-3p
}

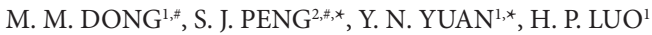 \\ ${ }^{1}$ Department of Gastroenterological Surgery, Central Hospital of Huangshi, Hubei, China; ${ }^{2}$ Intensive Care Unit, Yangxin People's Hospital, \\ Huangshi, Hubei, China
}

*Correspondence: Youneng_Yuan@163.com; 15072039995@qq.com

${ }^{\#}$ Contributed equally to this work.

Received September 27, 2018 / Accepted January 15, 2019

\begin{abstract}
Long noncoding RNAs (lncRNAs) were reported to participate in the progression of gastric cancer (GC). However, little is known about the biological functions of TTN antisense RNA 1 (TTN-AS1) in GC. Using qRT-PCR examination, we found that TTN-AS1 was expressed at a higher level in GC tissues and cell lines compared to the normal controls. KaplanMeier analysis of GC patients revealed the negative correlation between TTN-AS1 expression and the overall survival. To detect the biological function of TTN-AS1 in GC, we silenced TTN-AS1 to perform loss-of-function assays. The experimental results revealed that knockdown of TTN-AS1 obviously inhibited GC cell proliferation, induced cell apoptosis and impaired cell migration and invasion. In mechanism, TTN-AS1 was located in the cytoplasm of GC cells, indicating the post-transcriptional regulation of TTN-AS1 on gene expression. Bioinformatics analysis revealed the potential binding relation between TTN-AS1 and miR-376b-3p as well as between miR-376b-3p and KLF12. Mechanism experiments such as luciferase reporter assay and RNA pull-down assay demonstrated the interaction between TTN-AS1 and miR-376b-3p as well as between miR-376b-3p and KLF12 in GC cells. At last, rescue assays certified that miR-376b-3p and KLF12 involved in TTN-AS1-mediated GC progression. Similarly, the role of TTN-AS1-miR-376b-3p-KLF12 axis in GC progression was analyzed and validated. Taken together, we concluded that TTN-AS1 might function as a novel potential therapeutic target in the treatment of gastric cancer.
\end{abstract}

Key words: TTN-AS1, miR-376b-3p, KLF12, gastric cancer, ceRNA

Gastric cancer (GC) ranks as the fifth most common cancer $[1,2]$. In recent year, about 679100 new cases were diagnosed and the mortalities reached 498000 [3]. Metastasis and local recurrence are fatal causes for the poor overall 5 -years survival $[4,5]$. Therefore, exploring the effective diagnostic biomarkers is quite significant for the treatment of gastric cancer.

Long noncoding RNAs (lncRNAs) are defined as a subgroup of noncoding RNAs (ncRNAs) with more than 200 nucleotides [6]. In recent years, increasing number of reports suggested that lncRNAs were closely associated with tumor initiation and progression $[7,8]$. There are some lncRNAs that have been reported in gastric cancer. For instance, IncRNA NNT-AS1 promoted gastric cancer growth and differentiation via modulating miR-424/E2F1 axis [9]; long non-coding RNA RP11-789C1.1 inhibited gastric cancer; metastasis via regulating miR-5003/E-Cadherin axis [10]. LncRNA ZFPM2-AS1 accelerated gastric carcinogenesis by stabilizing MIF [11]. LncRNA TTN antisense RNA 1 (TTN-AS1) has been reported in cervical cancer [12] and esophageal squamous cell carcinoma [13]. Nevertheless, to our knowledge, the biological functions of TTN-AS1 in gastric cancer are still unknown.

MicroRNAs (miRNAs) were suggested to modulate the expression of their downstream targets post-transcriptionally [14]. Moreover, miRNAs can modulate various biological processes in human cancers, such as proliferation, migration and invasion $[15,16]$. Mechanistically, lncRNAs can act as competing endogenous RNAs (ceRNAs) to release mRNAs expression by sponging miRNAs [17, 18]. The lncRNAmiRNA-mRNA network was documented to modulate tumor initiation and development $[19,20]$. Therefore, we investigated whether TTN-AS1 can interact with a certain miRNA in GC cells. Bioinformatics analysis and mechanism investigation revealed the interaction between TTN-AS1 and miR-376b-3p. Similarly, the target of miR-376b-3p was 
predicted and searched out. Rescue assays were applied to demonstrate TTN-AS1-miR-376b-3p-KLF12 axis in GC. Since previous report has revealed the interaction between TTN-AS1 and miR-133b, we also performed experiments to demonstrate the role of TTN-AS1-miR-133b-FSCN1 pathway in GC. Collectively, this study demonstrated the ceRNA role of TTN-AS1 in gastric cancer.

\section{Materials and methods}

Tissues sample and culture. A total of 82 pairs of gastric cancer tissues and paired adjacent normal tissues were obtained from gastric patients at Central Hospital of Huangshi (Hebei, China). No patients were previously admitted for the treatment of chemotherapy or radiotherapy. An informed consent was received from all patients. This study was supported by The Ethics Committee of Central Hospital of Huangshi. All gastric cancer tissues were frozen in liquid nitrogen and stored at $-80^{\circ} \mathrm{C}$.

Cell lines and transfection. Four gastric cancer cell lines (MGC-803, MKN-45, BGC-823, SGC-7901) and one normal gastric mucosa cell line GES-1 were obtained from American Type Culture Collection (ATCC, Rockville, MD, USA). The cells were maintained in Dulbecco's Modified Eagle's medium (DMEM, Invitrogen, Carlsbad, CA, USA) containing 10\% fetal bovine serum (FBS; Gibco, Carlsbad, CA, USA) and $100 \mu \mathrm{g} / \mathrm{ml}$ streptomycin as well as $100 \mathrm{U} / \mathrm{ml}$ penicillin at $37^{\circ} \mathrm{C}$ with $5 \% \mathrm{CO}_{2}$.

Lipofectamine2000 (Invitrogen, USA) was utilized for transfection according to the manufacturer's protocol. Specific short hairpin RNA (shRNA) was used to target TTN-AS1 and then attenuated the expression of TTN-AS1 (sh-TTN-AS1) (GenePharma, Shanghai, China). Moreover, the miR-376b-3p mimics, miR-376b-3p inhibitor and their negative controls were purchased from GenePharma (Shanghai, China).

RNA extraction and qRT-PCR analysis. All RNAs were isolated from gastric cancer tissues and cell lines by TRIzol ${ }^{\circledR}$ reagent (Takara, Dalian, China) following the manufacturer's protocol. And cDNA was synthesized via a PrimeSxript RT reagent kit (Takara, Kusatsu, Shiga, Japan). The qRT-PCR was accomplished with a Bio-Rad CFX96 system (Bio-Rad, Foster City, CA, USA) in which a SYBR Premix ExTaq II kit (Takara) and mirVanaTM qRT-PCR miRNA Detection Kit (Ambion, Austin, TX, USA) were utilized to detect gene expression. The results were analyzed using the $2^{-\Delta \Delta C T}$ method. GAPDH and U6 functioned as the internal references.

Cell proliferation assays. In MTT assay, 3-(4,5-dimethylthiazol-2-yl)-2,5-diphenyltetrazolium bromide (MTT, Sigma, St. Louis, USA) was used here for the measurement of cell viability. Briefly, cells were seeded into 96-well plates ( $1 \times 10^{3}$ cells/well) for $1-3$ days. And then, $20 \mu \mathrm{l} 0.5 \mathrm{mg} / \mathrm{ml}$ MTT (Sigma, St. Louis, USA) was added to each well. After incubation, $200 \mu \mathrm{l}$ DMSO (Sigma, St. Louis, USA) was added to each well. Then, the absorbance of different cell lines at $560 \mathrm{~nm}$ was recorded with a microplate reader (Thermo, Rockford, USA).

In colony formation assay, cell lines were plated into 6-well plates (500 cells/well). After incubation for 12 days, cells were washed twice with cold PBS and fixed in methanol and thereby stained with $0.5 \%$ crystal violet. The number of cell colonies was visualized using a light microscope (Olympus, Tokyo, Japan).

Flow cytometry assay. Cell apoptosis was measured by Annexin V-Alexa Fluor 647/PI apoptosis detection kit (Fcmacs, Jiangsu, China). After digesting with collagenase, cell lines were collected and re-suspended within binding buffer. Then, cells were stained with Annexin V-Alexa Fluor 647 and Propidium Iodide and further cultured for $15 \mathrm{~min}$. The results were calculated by using a flow cytometer (BD FACS Aria; BD Biosciences, USA).

Transwell assay. The invasive or migratory ability was assessed using 24-well Transwells coated with or without $1 \mathrm{mg} / \mathrm{ml}$ Matrigel (Corning Co, USA). Transfected cell lines were seeded into the upper chamber with serum-free RPMI 1640. Moreover, $500 \mu \mathrm{l} 20 \%$ FBS medium was added to the lower chambers. Forty-eight hours later, cell lines that infiltrated to the bottom were fixed with $4 \%$ paraformaldehyde and stained with crystal violet for $24 \mathrm{~h}$. The number of invasive cells was counted in 5 randomly selected fields under a microscope (Thermo, Waltham, MA, USA).

Western blot analysis. Total protein was isolated from gastric cancer tissues and cell lines by RIPA lysis buffer (Beyotime, China). The concentration of proteins was analyzed by a BCA Protein assay kit (Beyotime). Then, protein extracts were subjected to $10 \%$ SDS-PAGE and then transferred into PVDF membranes (Thermo Fisher Scientific, USA). Subsequently, the membranes were blocked in $5 \%$ non-fat milk solution for $2 \mathrm{~h}$ and then incubated with antibodies including anti-E-cadherin (ab76055, Abcam, Cambridge, UK), anti-N-cadherin (ab76057, Abcam), anti-KLF12 (ab229828, Abcam), anti-active Caspase-3 (ab32042, Abcam), anti-Caspase-3 (ab4051, Abcam), antiactive Caspase-9 (ab2324, Abcam), Caspase-9 (ab52298, Abcam) and GAPDH (ab125247, Abcam) at $4^{\circ} \mathrm{C}$ overnight. Then the membranes were washed with PBST, followed by incubating with the appropriate secondary antibodies for $2 \mathrm{~h}$. GAPDH was regarded as an internal control. Proteins were visualized with an enhanced chemiluminescence (ECL) detection system (Millipore, MA, USA) and quantified using Image J software (NIH, Bethesda, MD, USA).

Subcellular fractionation. To conduct subcellular fractionation assay, PARIS ${ }^{\text {тм }}$ Kit was obtained commercially from Invitrogen (Carlsbad, CA, USA). MGC-803 and MKN-45 cells were cultured in cell fractionation buffer, followed by centrifugation. The nuclear and cytoplasmic fractions were isolated. After transferring cell supernatant to a fresh RNase-free tube, the lysates were rinsed with cell fractionation buffer, followed by centrifugation. Nuclei were 
Table 1. Correlation between the expression of TTN-AS1 and clinicopathological features of gastric cancer patients $(n=82)$.

\begin{tabular}{|c|c|c|c|}
\hline \multirow{2}{*}{ Variable } & \multicolumn{2}{|c|}{ TTN-AS1 expression } & \multirow{2}{*}{ p-value } \\
\hline & Low & High & \\
\hline \multicolumn{4}{|l|}{ Age } \\
\hline$<60$ & 12 & 14 & \multirow{2}{*}{0.636} \\
\hline$\geq 60$ & 30 & 26 & \\
\hline \multicolumn{4}{|l|}{ Gender } \\
\hline Male & 10 & 11 & \multirow{2}{*}{0.802} \\
\hline Female & 32 & 29 & \\
\hline \multicolumn{4}{|l|}{ Histological Grade } \\
\hline Well/Moderate & 19 & 17 & \multirow{2}{*}{0.827} \\
\hline Poor/Other & 23 & 23 & \\
\hline \multicolumn{4}{|l|}{ T Stage } \\
\hline $\mathrm{T} 1-\mathrm{T} 2$ & 27 & 10 & \multirow{2}{*}{$0.0004^{* * *}$} \\
\hline $\mathrm{T} 3-\mathrm{T} 4$ & 15 & 30 & \\
\hline \multicolumn{4}{|c|}{ Lymphatic Metastasis } \\
\hline Present & 28 & 13 & \multirow{2}{*}{$0.003^{*}$} \\
\hline Absent & 14 & 27 & \\
\hline \multicolumn{4}{|l|}{ TNM Stage } \\
\hline I-II & 25 & 13 & \multirow{2}{*}{$0.016^{*}$} \\
\hline III-IV & 17 & 27 & \\
\hline
\end{tabular}

Low/high decided by the sample mean. Pearson $\chi^{2}$ test. ${ }^{*} \mathrm{p}<0.05,{ }^{* *} \mathrm{p}<0.01$, ${ }^{* * *} \mathrm{p}<0.001$ was considered statistically significant.

acquired using cell disruption buffer. The aforementioned cell supernatant and lysates were incubated with $2 \times$ lysis/ binding solution and equal volume of ethanol. Finally, all samples were rinsed in PBS. The expression of TTN-AS1, GAPDH (cytoplasm control) and U6 (nucleus control) was analyzed by qRT-PCR.

Dual luciferase reporter assay. The fragment of TTN-AS1 containing putative binding sites for miR-376b-3p was predicted. Gastric cancer cells were co-transfected with pmirGLO-TTN-AS1-WT or pmirGLO-TTN-AS1-MUT reporter plasmids as well as miR-376b-3p mimics or miR-NC. After $24 \mathrm{~h}$ transfection, luciferase activity was detected by the dual luciferase assay kit (Promega, Madison, WI, USA) and the results were normalized to Renilla activity.

RNA immunoprecipitation (RIP) assay. RIP assay was carried out using EZ-Magna RIP kit (Millipore, Billerica, MA) following the instruction of manufacturers. MGC-803 and MKN-45 cells were lysed with complete RIP lysis buffer and then incubated with the RIP buffer, which containing magnetic beads with anti-Ago2 or anti-IgG antibodies (Millipore, USA). IgG (Millipore, USA) was utilized as a negative control. After incubation for $2 \mathrm{~h}$ at $4{ }^{\circ} \mathrm{C}$, the enrichment of TTN-AS1 in the immunoprecipitated RNA was analyzed by qRT-PCR.

RNA pull-down assay. RNA pull-down assays were carried out by Magnetic RNA-Protein Pull-Down Kit (Pierce, USA) following the manufacturer's instructions. In brief, miR-376b-3p-WT, miR-376b-3p-MUT and miR-NC were respectively biotinylated to be bio-miR-376b-3p-WT, bio-miR-376b-3p-MUT and bio-miR-NC via GenePharma Company (Shanghai, China). Then, these miRNAs were transfected into MGC-803 and MKN-45 cells. After transfection for $48 \mathrm{~h}$, two cell lines were harvested. The enrichment of KLF12 was detected by RT-PCR.

Statistical analysis. All data are shown as the mean \pm standard deviation (SD). SPSS software deviation 17.0 (IBM Corporation, Armonk, NY, USA) was used for statistical analysis. Survival curves were generated and analyzed using the Kaplan-Meier method and log-rank test. The comparisons between groups were detected by the Student's t-test and one-way analysis of variance (ANOVA). Expression correlations between TTN-AS1 and miR-376b-3p or KLF12 were evaluated by Spearman's correlation analysis. $p$ value less than 0.05 was considered statistically significant.

\section{Results}

TTN-AS1 was significantly upregulated in gastric cancer tissues and cell lines. To assess the specific role of TTN-AS1 in gastric cancer, we examined the expression of TTN-AS1 in gastric cancer tissues by qRT-PCR. As displayed in Figure 1A, TTN-AS1 was significantly upregulated in gastric cancer tissues compared with adjacent normal tissues. Moreover, we also found that the expression of TTN-AS1 was obviously increased in four gastric cancer cell lines (MGC-803, MKN-45, BGC-823, SGC-7901), especially in MGC-803 and MKN-45 cell lines (Figure 1B). In order to verify the association between TTN-AS1 expression and clinico-pathological characteristics, 82 GC samples were stratified into two groups according to the mean value of TTN-AS1 expression. As shown in Table 1, TTN-AS1 expression was closely related to T Stage, lymphatic metastasis and TNM Stage. The overall survival (OS) curve was generated to further investigate the potential prognostic value of TTN-AS1 for GC patients. The results demonstrated that high expression of TTN-AS1 was closely associated with the poor overall survival of patients with GC (Figure 1C). Taken together, our findings indicated that TTN-AS1 might be a potential factor contributed to the poor prognosis of GC patients.

Silencing of TTN-AS1 inhibited cell proliferation and facilitated cell apoptosis in gastric cancer. Considering that TTN-AS1 was expressed highest in MGC-803 and MKN-45 cell lines, we then silenced TTN-AS1 expression in MGC-803 and MKN-45 cell lines via transfecting sh-TTN-AS1 (Figure 2A). Then MTT assay and colony formation assay showed that TTN-AS1 knockdown remarkably suppressed cell viability and proliferation in MGC-803 and MKN-45 cells (Figure 2B, 2C). Flow cytometry assay demonstrated that inhibition of TTN-AS1 obviously increased the rate of apoptosis in MGC-803 and MKN-45 cells (Figure 2D). More importantly, western blot assay demonstrated that silencing of TTN-AS1 increased the protein level of cleaved caspase 3 and cleaved caspase 
A

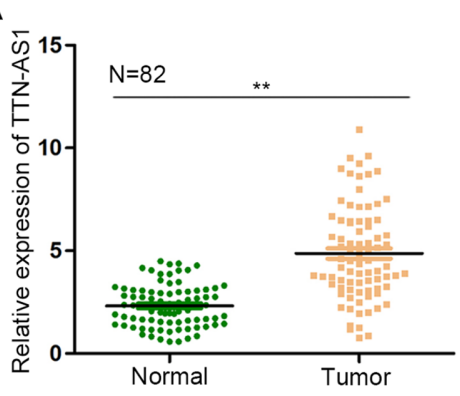

B

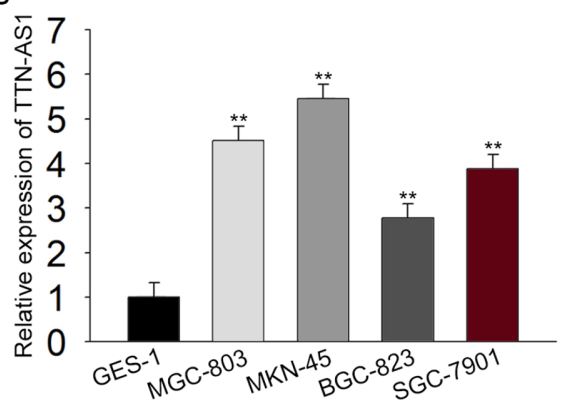

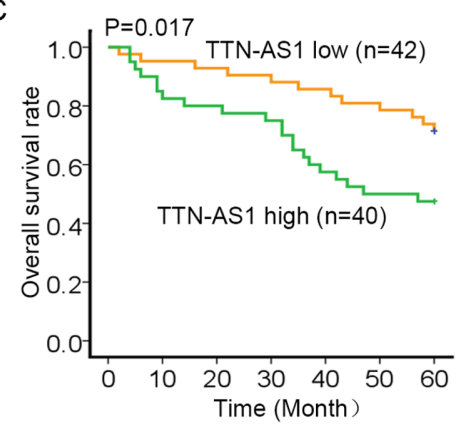

Figure 1. TTN-AS1 was significantly upregulated in gastric cancer tissues and cell lines. A) The expression of TTN-AS1 in gastric cancer tissues and adjacent normal tissues was observed by qRT-PCR. B) qRT-PCR measured the TTN-AS1 expression in four gastric cancer cell lines (MGC-803, MKN45, BGC-823, SGC-7901) and normal gastric mucosa cell line GES-1. C) Kaplan-Meier method and log-rank test showed the overall survival rate of gastric cancer patients with high or low TTN-AS1 expression. ${ }^{*} \mathrm{p}<0.05,{ }^{* *} \mathrm{p}<0.01$.

A

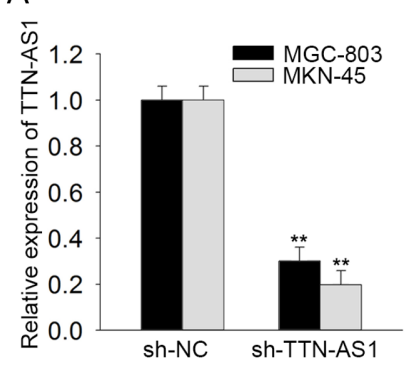

B

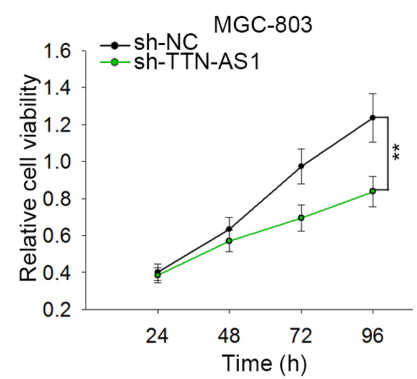

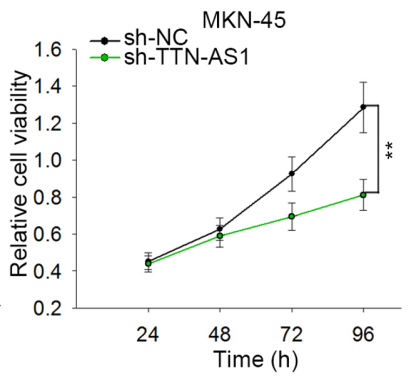

E

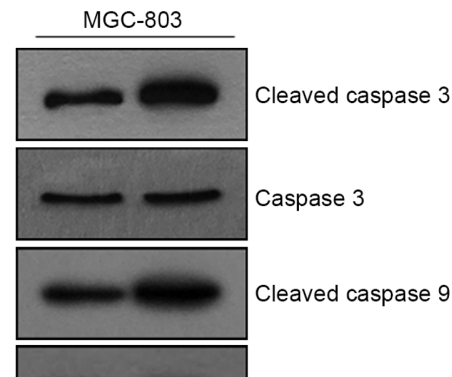

Caspase 9

C
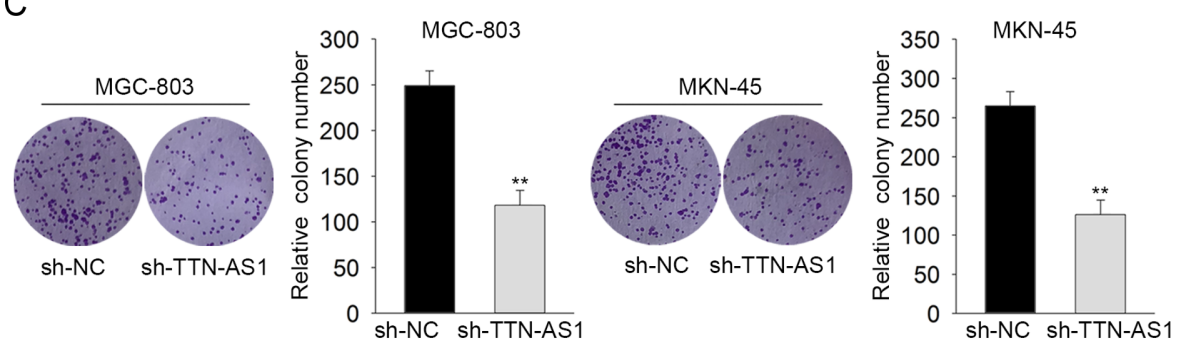

D
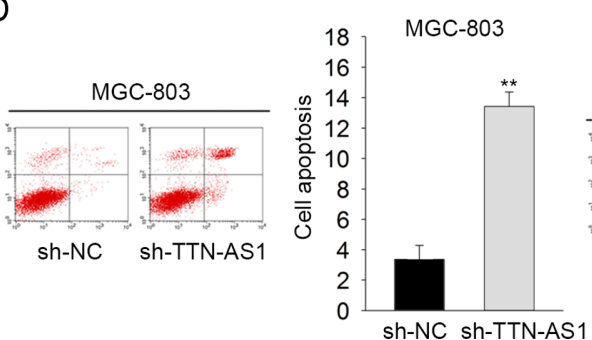

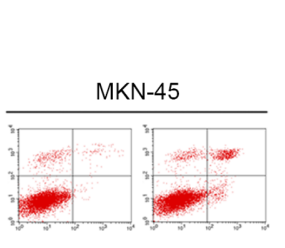

sh-NC sh-TTN-AS1

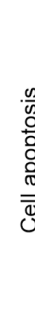

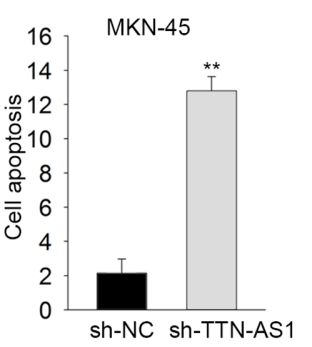
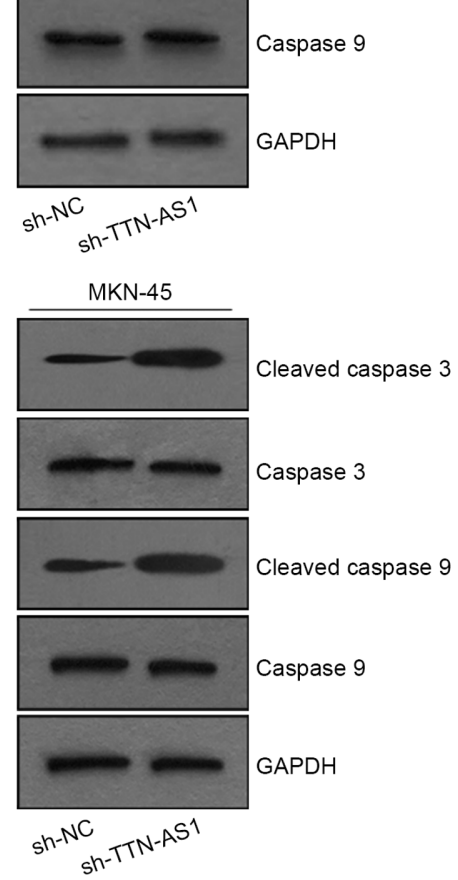

Figure 2. Silencing of TTN-AS1 inhibited cell proliferation and facilitated apoptosis in gastric cancer. A) qRT-PCR assay was utilized to investigate the expression level of TTN-AS1 in MGC-803 and MKN-45 cells transfected with sh-TTN-AS1 or sh-NC. B-C) The effect of TTN-AS1 knockdown on MGC-803 and MKN-45 cells proliferation was detected by MTT assay and colony formation assay. D) Flow cytometry assay revealed the cell apoptosis in MGC-803 and MKN-45 cells transfected with sh-TTN-AS1. E) Western blot analysis showed the protein levels of total caspase 3, cleaved caspase 3, total caspase 9 and cleaved caspase 9 in MGC-803 and MKN-45 cell lines. ${ }^{*} \mathrm{p}<0.05,{ }^{* *} \mathrm{p}<0.01$. 
9, but did not obviously change total caspase 3 and total caspase 9 (Figure 2E), indicating the positive effect of TTN-AS1 knockdown on GC cell apoptosis.

Knockdown of TTN-AS1 repressed cell migration and invasion in gastric cancer. In addition, the effect of TTN-AS1 knockdown on cell migration and invasion was analyzed by transwell assay. We found that knockdown of TTN-AS1 dramatically reduced the cell migration and invasion in both MGC-803 and MKN-45 cell lines (Figures 3A, 3B). Furthermore, western blot assay confirmed that TTN-AS1 knockdown increased the protein level of $\mathrm{N}$-cadherin, while decreased $\mathrm{E}$-cadherin protein level in MGC-803 and MKN-45 cells (Figure 3C). These results elucidated that TTN-AS1 played an oncogenic function in gastric cancer.

TTN-AS1 acted as a molecular sponge of miR-376b-3p in gastric cancer. Increasing evidences have revealed that lncRNAs can exert its biological functions in human cancers through sponging miRNAs $[21,22]$. In the present study, we determined the cytoplasmic localization of TTN-AS1 in GC cells (Figure 4A), indicating the post-transcriptional regulatory role of TTN-AS1. Searching from starBase database (http://starbase.sysu.edu.cn/index.php), miR-376b-3p harbored the binding sites with TTN-AS1. In contrast to TTN-AS1, miR-376b-3p was expressed at a low level in GC tissues or cell lines (Figures 4B, 4C). According to Spearman's correlation analysis, the expression level of miR-376b-3p in GC tissues was negatively related with that of TTN-AS1 (Figure 4D). As presented in Figure 4E, the putative binding sequence of miR-376b-3p and TTN-AS1 was predicted. Luciferase reporter assay demonstrated that miR-376b-3p mimics obviously decreased the luciferase activity of wild type TTN-AS1 (TTN-AS1-WT). However, no significant change was observed in mutant TTN-AS1 (TTN-AS1-MUT) in both MGC-803 and MKN-45 cells (Figure 4F). RIP assay further confirmed that TTN-AS1 and miR-376b-3p were abundant in Ago2 pellet, indicating that they were involved in the same RISC complex (Figure 4G). In addition, we also
A

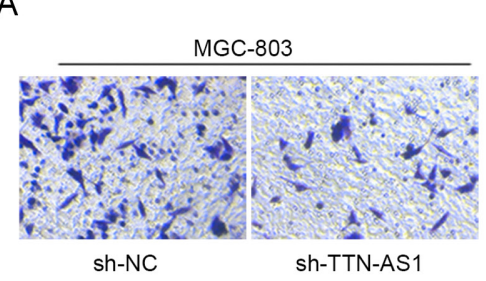

B

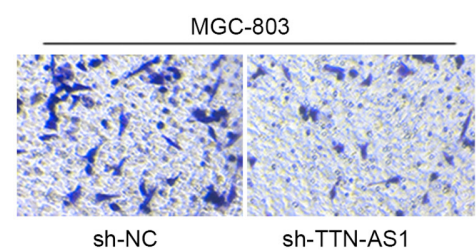

C

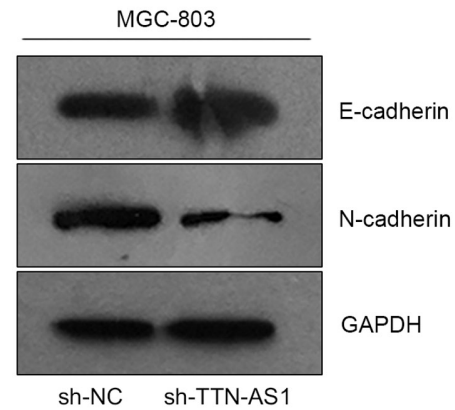

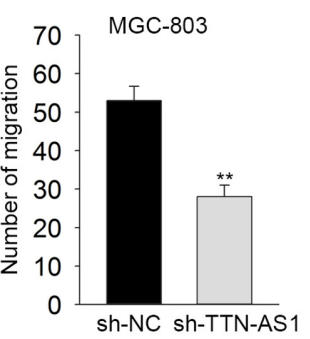
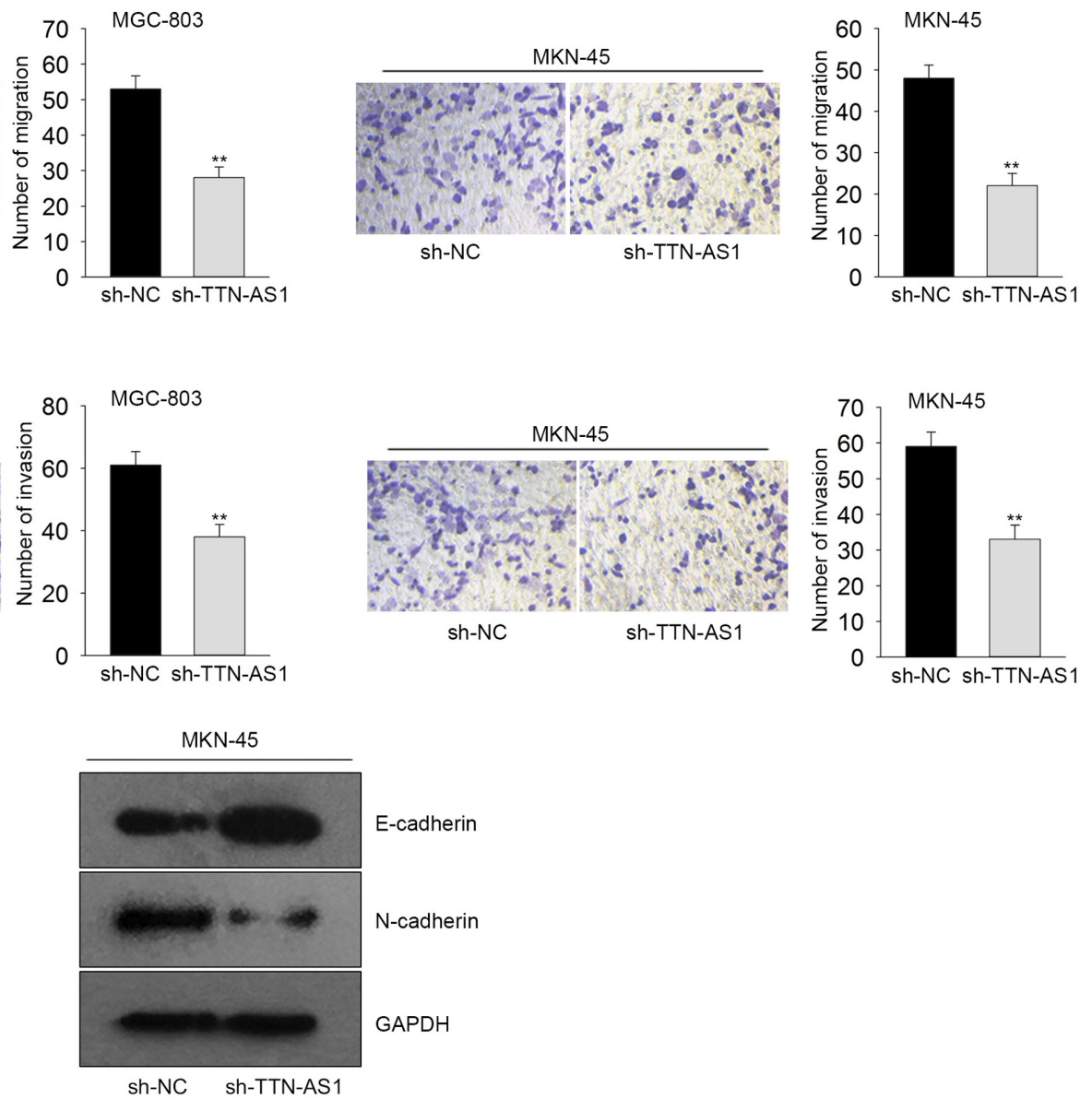

Figure 3. Knockdown of TTN-AS1 repressed cell migration and invasion in gastric cancer. A-B) The migratory and invasive abilities of MGC-803 and MKN-45 cells were detected by transwell assay after transfection with sh-TTN-AS1 or sh-NC. C) The protein levels of EMT-related markers in MGC803 and MKN-45 cells after transfection of sh-TTN-AS1 or sh-NC were investigated by western blot assay. GAPDH was utilized as internal control. ${ }^{*} \mathbf{p}<0.05,{ }^{* *} \mathbf{p}<0.01$. 
found that TTN-AS1 knockdown remarkably elevated the expression of miR-376b-3p in MGC-803 and MKN-45 cells (Figure 4H).

KLF12 is a target gene of miR-376b-3p in gastric cancer. We then explored the potential targets of miR-376b-3p using four bioinformatics tools (PITA, PicTar, TargetScan and microT). 38 putative targets of miR-376b-3p were searched out (Figure 5A). The expression levels of all these 38 mRNAs were measured in response to the knockdown of TTN-AS1 or the upregulation of miR-376b-3p. As shown in Figure 5B, KLF12 was significantly downregulated in cells transfected with sh-TTN-AS1 and miR-376b-3p. Moreover, KLF12 has been reported to be a positive regulator in gastric cancer progression [23]. Therefore, we chose KLF12 for further analysis. We obtained the binding sites between miR-376b-3p and KLF12 from TargetScan (http://www.targetscan.org/ vert_72/) (Figure 5C). Based on the luciferase activity analysis, miR-376b-3p mimics dramatically reduced the luciferase activity in wild type KLF12 (KLF12-WT) but not changed that of mutant KLF12 (KLF12-MUT) (Figure 5D). RNA pull-down assay further confirmed the interaction of miR-376b-3p and KLF12 (Figure 5E). Then qRT-PCR assay uncovered that KLF12 was highly expressed in gastric cancer tissues and cell line (Figures 5F, 5G). Furthermore, Spearman's correlation analysis revealed the negative association between KLF12 and miR-376b-3p (Figure 5H) as well as the positive association between KLF12 and TTN-AS1 (Figure 5I).
A

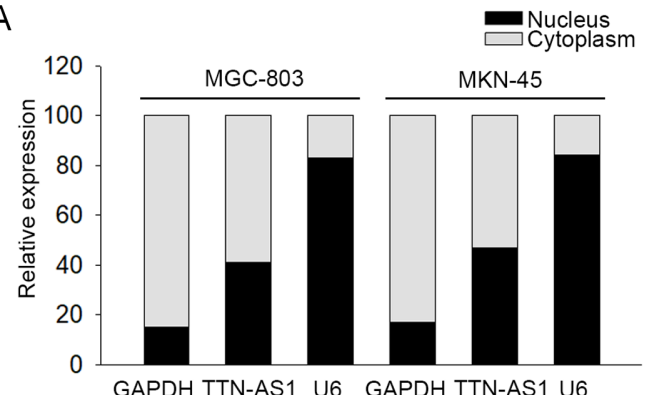

D
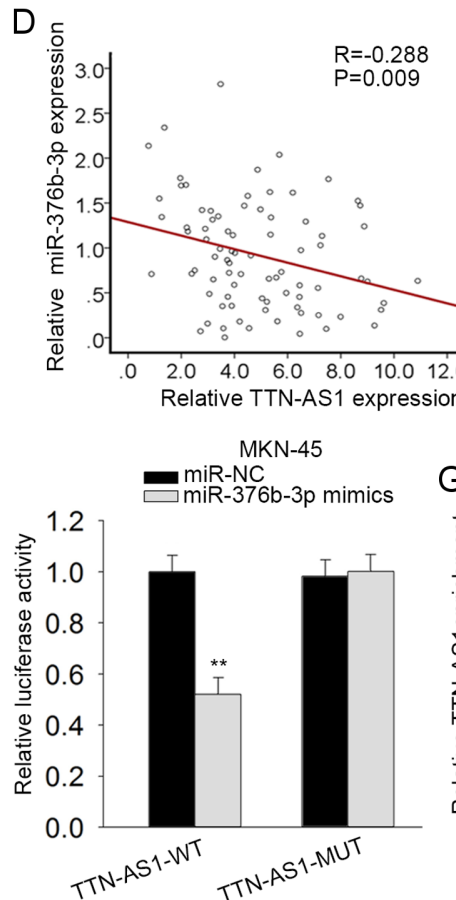

$E$
$B$

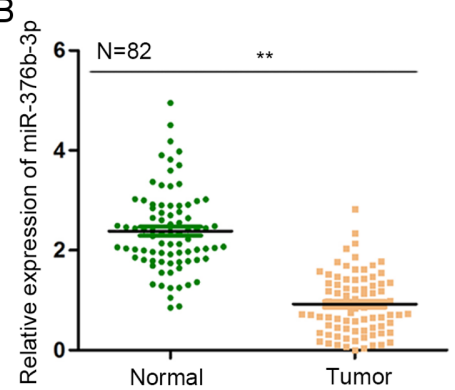

C

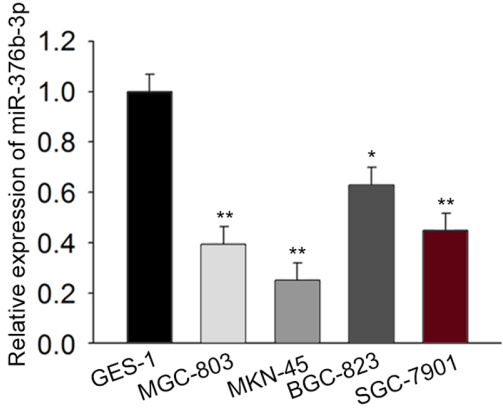

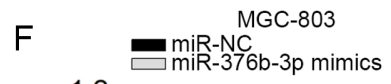
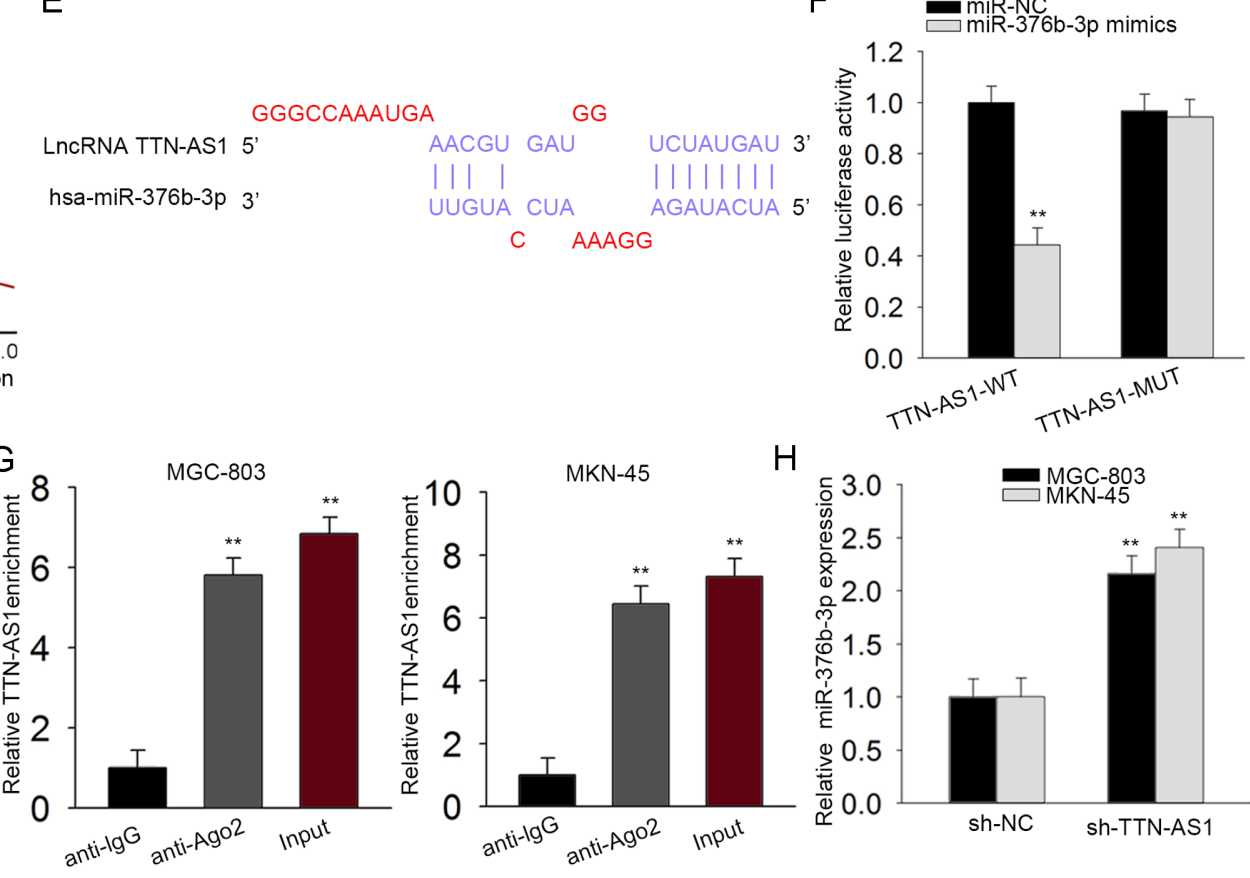

$\mathrm{H}$

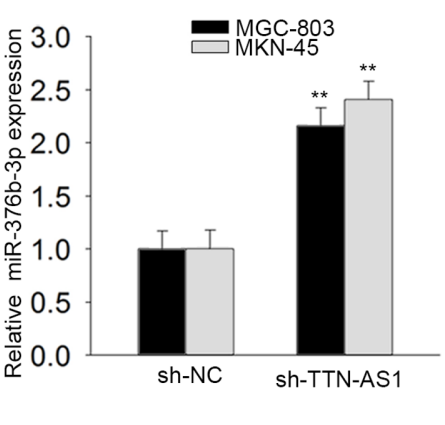

Figure 4. TTN-AS1 acted as a molecular sponge of miR-376b-3p in gastric cancer. A) The cellular localization of TTN-AS1 was determined by subcellular fractionation assay. GAPDH: cytoplasmic control, U6: nuclear control. B-C) miR-376b-3p expression was explored in GC tissues and cell lines using qRT-PCR assay. D) Spearman's correlation analysis showed the correlation between TTN-AS1 expression and miR-376b-3p expression. E) The binding sites between TTN-AS1 and miR-376b-3p were obtained. F) Luciferase reporter assay revealed the binding relation between TTN-AS1 and miR-376b-3p. G) RIP assay confirmed the interaction between TTN-AS1 and miR-376b-3p. H) mRNA level of miR-376b-3p after TTN-AS1 knockdown was observed by $\mathrm{qRT}-\mathrm{PCR}$ assay. ${ }^{*} \mathrm{p}<0.05,{ }^{* *} \mathrm{p}<0.01$. 
A

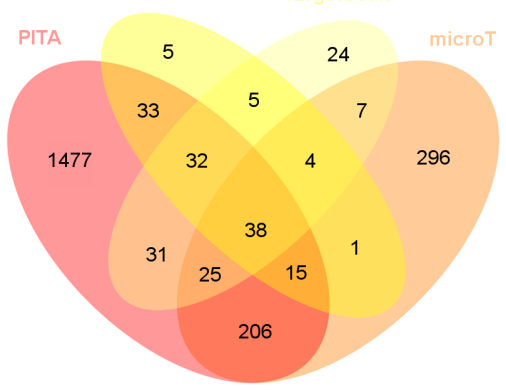

B

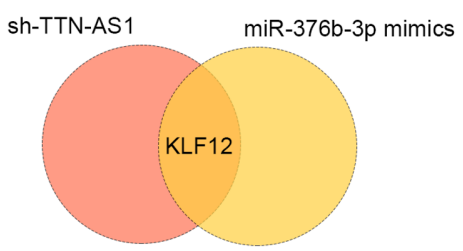

C

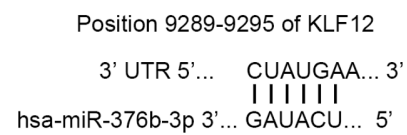

D

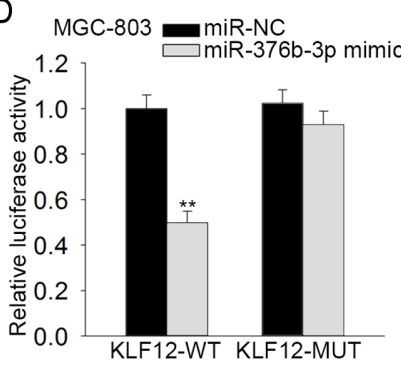

G

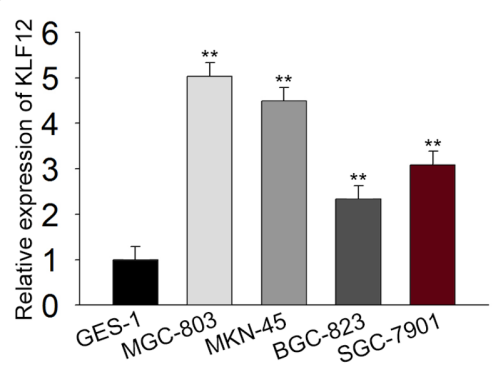

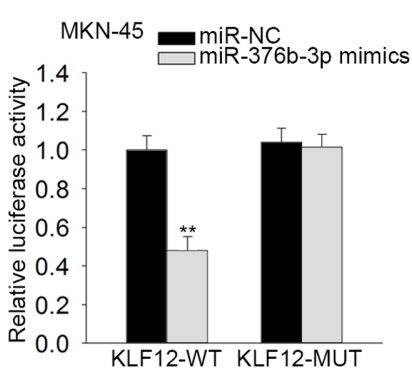

$E$

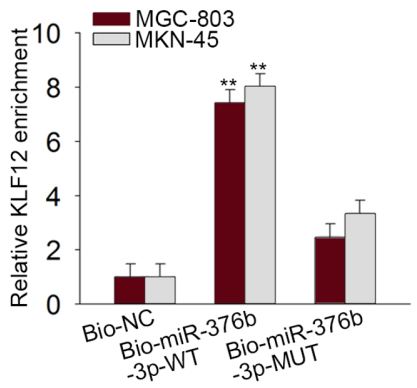

1

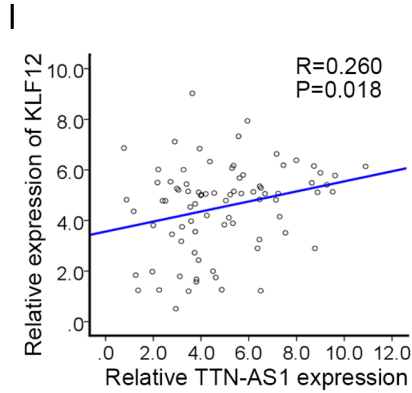

Figure 5. KLF12 is a target gene of miR-376b-3p in gastric cancer. A) The putative targets of miR-376b-3p were predicted using four bioinformatics tools (PITA, PicTar, TargetScan and microT). B) KLF12 was significantly downregulated in response to the knockdown of TTN-AS1 or the upregulation of miR-376b-3p. C) The putative binding sites of miR-376b-3p in KLF12 3'UTR were shown. D) The interaction between miR-376b-3p and KLF12 in MGC-803 and MKN-45 cells was certified by luciferase reporter assay. E) MGC-803 and MKN-45 cells were transfected with biotinylated wild type miR-376b-3p (bio-miR-376b-3p-WT) or mutant type miR-376b-3p (bio-miR-376b-3p-MUT) to confirm the interaction between miR-376b-3p and KLF12 by RNA pull-down assay. F-G) The expression level of KLF12 in gastric cancer tissues and cell lines was examined by qRT-PCR assay. H-I) Spearman's correlation analysis revealed the correlation between KLF12 expression and miR-376b-3p expression or TTN-AS1 expression in GC tissues. ${ }^{*} \mathrm{p}<0.05,{ }^{* *} \mathrm{p}<0.01$.

TTN-AS1/miR-376b-3p/KLF12 axis facilitated cell proliferation and migration in gastric cancer. The results of qRT-PCR and western blot assay demonstrated that miR-376b-3p mimics significantly reduced the mRNA level and protein levels of KLF12, but the effects were reversed by co-transfection with pcDNA-TTN-AS1 (Figures 6A, 6B). To explore the effect of TTN-AS1/miR-376b-3p/KLF12 axis on cell proliferation and invasion in gastric cancer, rescue assays were conducted in MGC-803 cell line. As for cell proliferation, the inhibitory effect of sh-TTN-AS1 on the GC cell proliferation was attenuated by co-transfection with miR-376b-3p inhibitor or pcDNA-KLF12 (Figures 6C, 6D). Additionally, co-transfection with miR-376b-3p inhibitor or
pcDNA-KLF12 remarkably restored the cell migration and invasion caused by sh-TTN-AS1 (Figures 6E, 6F). Taken together, these results suggested that TTN-AS1 contributed to $\mathrm{GC}$ progression via modulating miR-376b-3p/KLF12 axis.

TTN-AS1 led to the upregulation of FSCN1 by sponging miR-133b. LncRNA TTN-AS1 has been previously shown to sponge miR-133b in esophageal cancer [13]. Considering some degree of similarity between the two organs of gastrointestinal system, we further ascertained TTN-AS1/ miR-133b/FSCN1 pathway in gastric cancer. At first, we measured the relative expression of miR-133b and FSCN1 in GC tissues. Unsurprisingly, miR-133b was expressed lower in GC tissues (Figure 7A), which was opposite with that of 
A

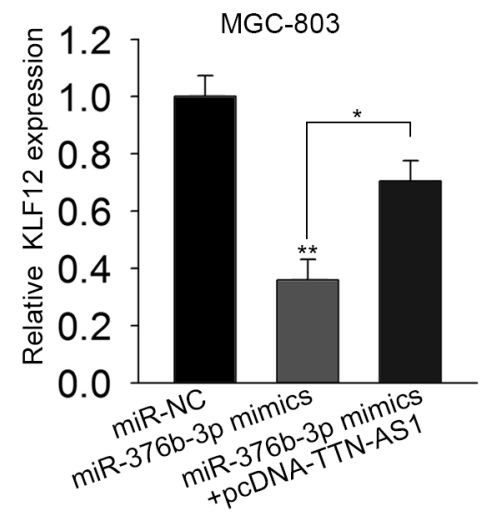

C

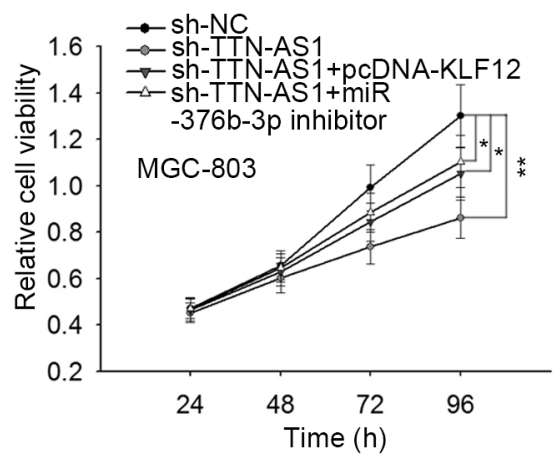

E

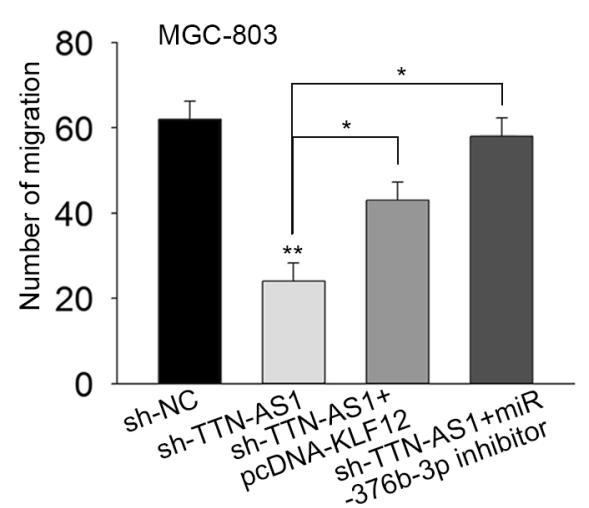

B

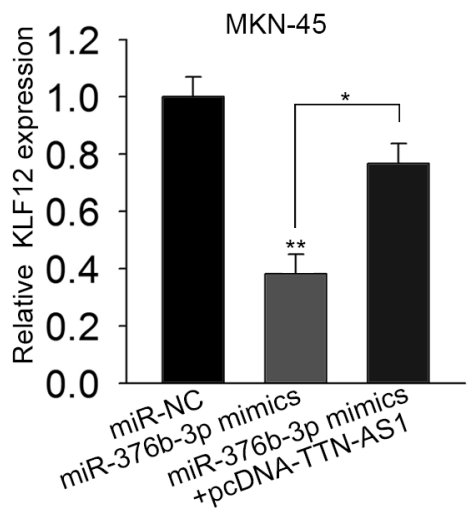

D

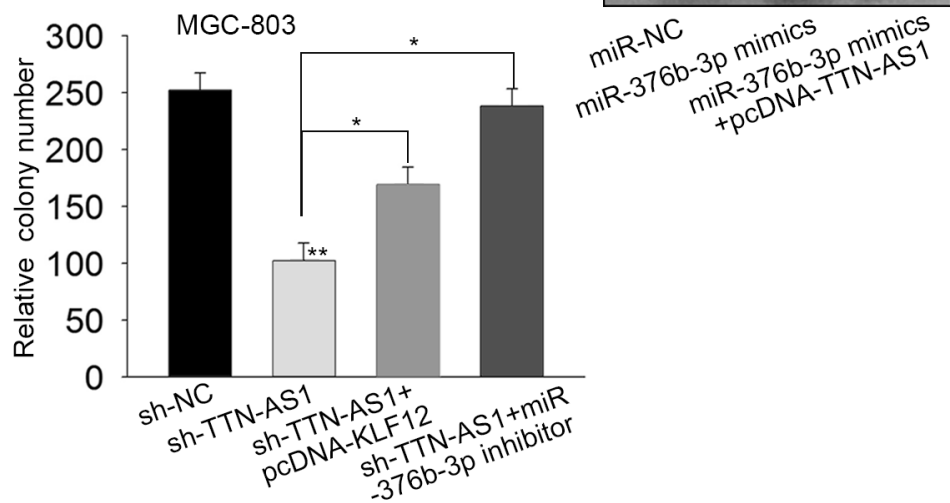

F

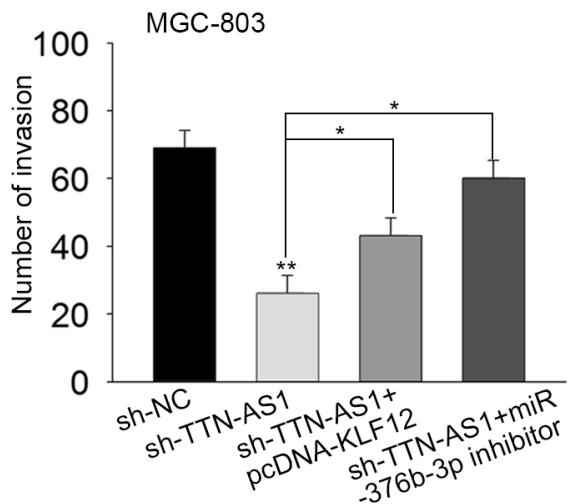

Figure 6. TTN-AS1/miR-376b-3p/KLF12 axis facilitated cell proliferation and migration in gastric cancer. A-B) qRT-PCR and western blot assay were performed to examine the mRNA level and protein level of KLF12 in MGC-803 and MKN-45 cells after transfection of miR-376b-3p mimics or co-transfection of miR-376b-3p mimics and pcDNA-TTN-AS1. C-D) Cell proliferation in TTN-AS1-downreguated MGC-803 cell was examined after co-transfection with miR-376b-3p inhibitor or sh-TTN-AS1 or pcDNA-KLF12. E-F) Transwell assay was performed to examine the migratory and invasive ability of TTN-AS1-downregulated MGC-803 cell after co-transfection with miR-376b-3p inhibitor or sh-TTN-AS1 and pcDNA-KLF12. ${ }^{*} \mathrm{p}<0.05,{ }^{* *} \mathrm{p}<0.01$.

FSCN1 (Figure 7B). The association between TTN-AS1 and FSCN1 was found to be positive in GC tissues. Bioinformatics analysis revealed that miR-133b harbored the binding sites in TTN-AS1 and FSCN1 sequence (Figure 7C). Similarly, luciferase activity analysis indicated the decreased luciferase activity of TTN-AS1-WT in GC cells transfected with miR-133b mimics (Figure 7D). However, this inhibition was partially reversed by the co-transfection of pcDNATTN-AS1. At last, the mRNA and protein levels of FSCN1 were detected in cells transfected with miR-133b mimics or 


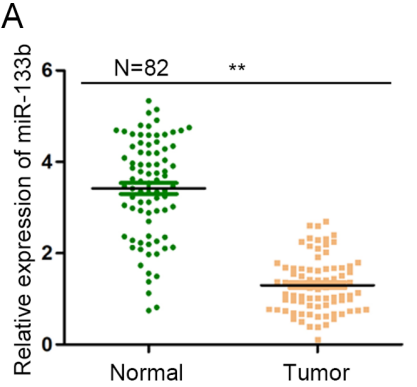

C

TtN-AS1: 5' CTGgtgaAataAaAgGaccaAa 3' || || |||| miR-133b: 3' AUCGACCAACUUCCCCUGGUUU 5' || ||||
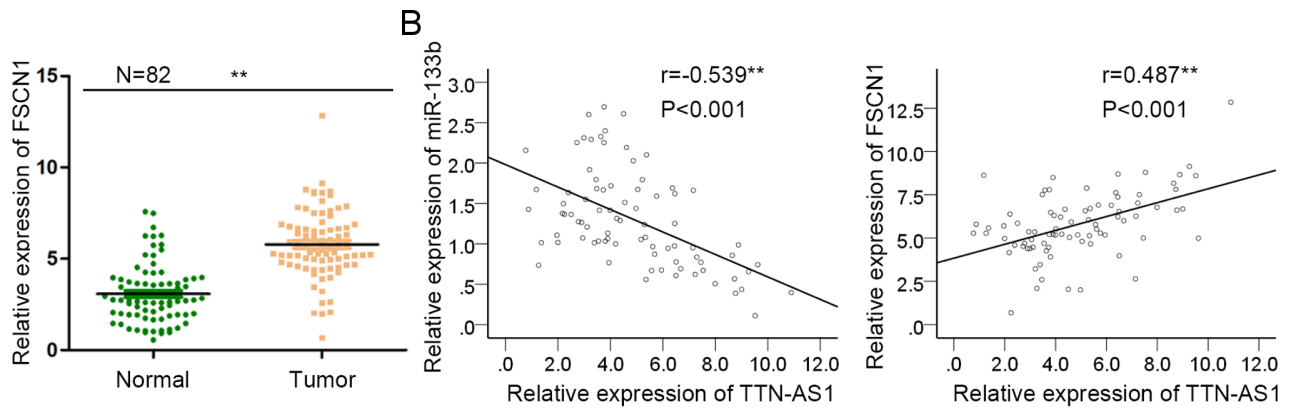
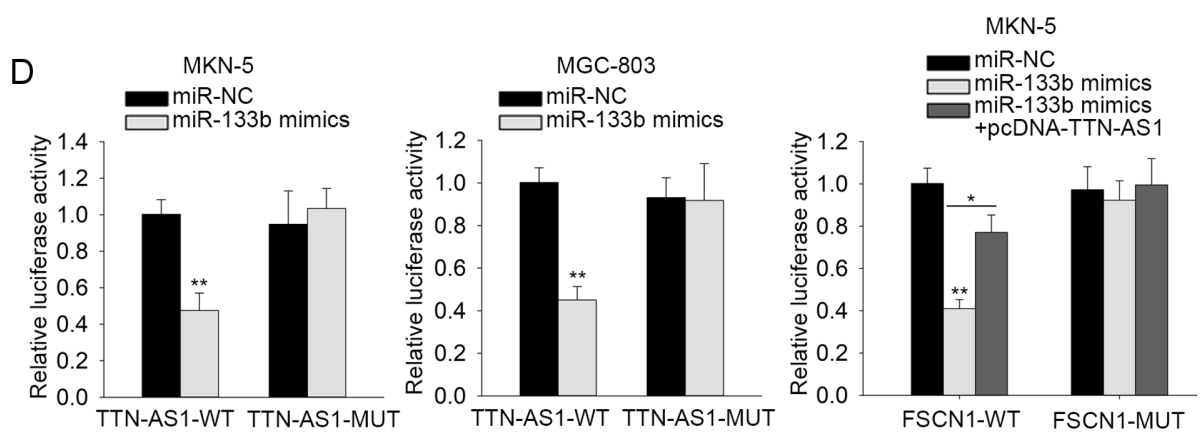
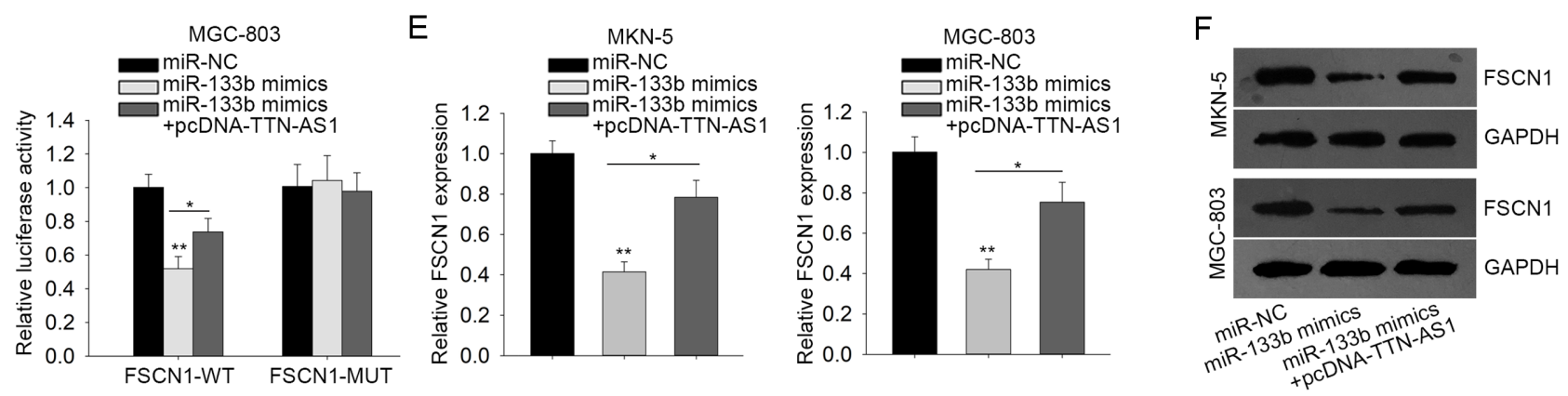

Figure 7. TTN-AS1 led to the upregulation of FSCN1 by sponging miR-133b. A). The expression of miR-133b and FSCN1 in GC tissues was examined. B) Correlation analysis of the TTN-AS1 expression and miR-133b expression or FSCN1 expression. C) miR-133b harbored the binding sites in TTNAS1 and FSCN1 sequence. D) Luciferase activity analysis of TTN-AS1-WT vector or TTN-AS1-MUT vector in GC cells transfected with miR-133b mimics or miR-NC. E-F) mRNA and protein levels of FSCN1 in GC cells transfected with miR-133b mimics or co-transfected with miR-133b mimics and pcDNA-TTN-AS1. ${ }^{*} \mathrm{p}<0.05,{ }^{* *} \mathrm{p}<0.01$.

co-transfection with miR-133b-3p and pcDNA-TTN-AS1. The results demonstrated that the inhibitory effect of miR-133b mimics on the FSCN1 expression was partly recovered by the co-transfection of pcDNA-TTN-AS1 (Figures 7E, 7F).

TTN-AS1/miR-133b/FSCN1 axis regulated GC cell proliferation and migration. We applied rescue assays to reveal the role of TTN-AS1/miR-133b/FSCN1 axis in regulating GC cell proliferation and migration. As illustrated in Figure $8 \mathrm{~A}$ and Figure $8 \mathrm{~B}$, cell viability and cell proliferation suppressed by the transfection of sh-TTN-AS1 were partly reversed by the introduction of pcDNA-FSCN1 or miR-133b inhibitor. Additionally, the inhibitory influences of silenced TTN-AS1 on GC cell migration and invasion were attenuated by the overexpression of FSCN1 or the inhibition of miR-133b (Figures 8C, 8D). These data suggested that TTN-AS1 regulated miR-133b/FSCN1 axis to modulate GC progression.

\section{Discussion}

Increasing evidences have revealed the vital role of lncRNAs in cancer progression, including initiation and differentiation [24-26]. Recently, the role of lncRNAs in gastric cancer has been studied $[27,28]$. LncRNA TTN-AS1 was previously suggested to be highly expressed in cervical cancer and esophageal squamous cell carcinoma and promoted tumor progression $[12,13]$. Nevertheless, the role of TTN-AS1 in gastric cancer is almost unknown. This 

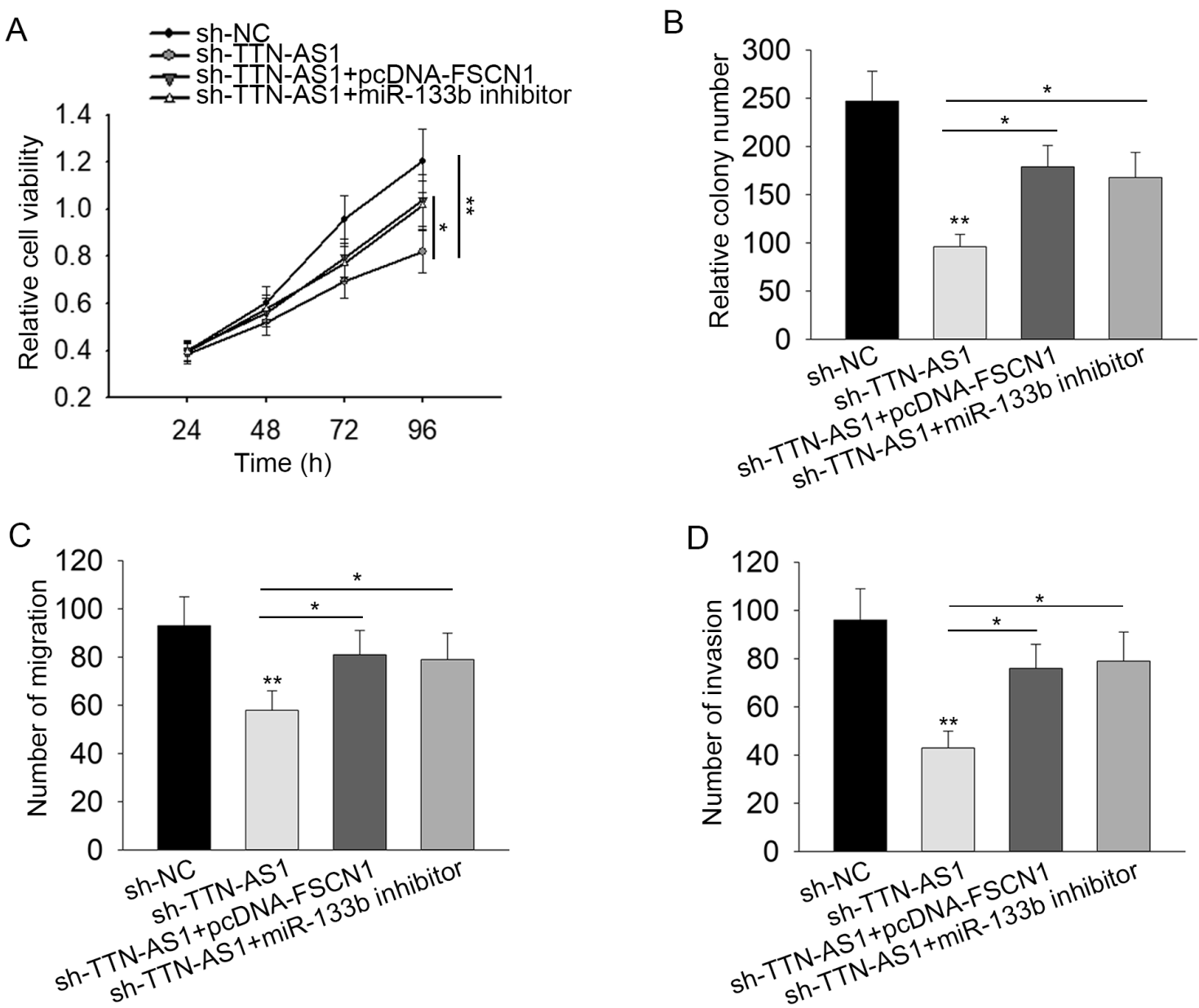

Figure 8. TTN-AS1/miR-133b/FSCN1 axis regulated GC cell proliferation and migration. A-B) Cell viability and cell proliferation suppressed by the transfection of sh-TTN-AS1 were partly reversed by the introduction of pcDNA-FSCN1 or miR-133b inhibitor. C-D) The migratory and invasive ability of TTN-AS1-downregulated GC cell was examined after co-transfection of miR-133b inhibitor or pcDNA-FSCN1. ${ }^{*} \mathrm{p}<0.05,{ }^{* *} \mathrm{p}<0.01$.

study focused on the function and potential mechanism of TTN-AS1 in GC progression. After qRT-PCR analysis, we identified the upregulation of TTN-AS1 in GC samples. Based on the clinical analysis, the association between TTN-AS1 expression and T Stage, lymphatic metastasis or TNM Stage was determined. Previous reports have focused on the prognostic value of lncRNAs in cancer patients [29, 30]. It is unknown that whether TTN-AS1 can affect the prognosis of GC patients. Our current study revealed the negative association between TTN-AS1 expression and the overall survival rate of GC patients. Therefore, we confirmed the research value of TTN-AS1 in gastric cancer. It is necessary to detect the role of TTN-AS1 in biological processes of GC. In this regard, loss-of-function assays were carried out. Interestingly, knockdown of TTN-AS1 efficiently suppressed cell proliferation, accelerated cell apoptosis and inhibited cell migration and EMT progress. Thus, we identified the oncogenic role of TTN-AS1 in GC progression.
Various reports have indicated the predominant role of lncRNAs in a ceRNA network [31-33]. According to the previous studies, TTN-AS1 regulates the progression of cervical cancer and esophageal squamous cell carcinoma via modulation of miRNA-mRNA axis. In the present study, we also detected whether the previous network was functional in gastric cancer. Mechanism investigation revealed that TTN-AS1 was predominantly located in the cytoplasm of GC cells and post-transcriptionally regulated miR-133b-FSCN1 axis in GC. Similarly, the novel molecular mechanism of TTN-AS1 was analyzed and demonstrated. It was found that TTN-AS1 acted as the miR-376b-3p sponge in GC to upregulate KLF12. Finally, the function of TTN-AS1-miR376b-3p-KLF12 axis or TTN-AS1-miR-133b-FSCN1 axis in GC progression was demonstrated by rescue assays. Interestingly, miR-376b-3p and KLF12 reversed TTN-AS1-mediated function. Also miR-133b and KLF12 partly reversed the function of TTN-AS1 in GC. Therefore, we confirmed that 
TTN-AS1 exerted oncogenic function in GC via modulation of miR-376b-3p-KLF12 axis or miR-133b-FSCN1 axis. In conclusion, TTN-AS1 was upregulated in GC and possibly associated with the poor prognosis of GC patients. Mechanistically, TTN-AS1 post-transcriptionally regulated FSCN1 and KLF12 by sponging miR-133b and miR-376b-3p. All our findings might contribute to exploring the novel diagnostic or therapeutic targets for GC patients.

Acknowledgements: We expressed our thanks to all patients.

\section{References}

[1] JI C, ZHAO Y, KOU YW, SHAO H, GUO L et al. Cathepsin F Knockdown Induces Proliferation and Inhibits Apoptosis in Gastric Cancer Cells. Oncol Res 2018; 26: 83-93. https:// doi.org/10.3727/096504017X14928634401204

[2] ALLUM W, LORDICK F, ALSINA M, ANDRITSCH E, BASSALAMAH A et al. ECCO essential requirements for quality cancer care: Oesophageal and gastric cancer. Crit Rev Oncol Hematol 2018; 122: 179-193. https://doi.org/10.1016/j. critrevonc.2017.12.019

[3] CHEN W, ZHENG R, BAADE PD, ZHANG S, ZENG H et al. Cancer statistics in China, 2015. CA Cancer J Clin 2016; 66: 115-132. https://doi.org/10.3322/caac.21338

[4] SITARZ R, SKIERUCHA M, MIELKO J, OFFERHAUS GJA, MACIEJEWSKI R et al. Gastric cancer: epidemiology, prevention, classification, and treatment. Cancer Manag Res 2018; 10: 239-248. https://doi.org/10.2147/CMAR.S149619

[5] KARIMI P, ISLAMI F, ANANDASABAPATHY S, FREEDMAN ND, KAMANGAR F. Gastric cancer: descriptive epidemiology, risk factors, screening, and prevention. Cancer Epidemiol Biomarkers Prev 2014; 23: 700-713. https://doi. org/10.1158/1055-9965.EPI-13-1057

[6] FATICA A, BOZZONI I. Long non-coding RNAs: new players in cell differentiation and development. Nat Rev Genet 2014; 15: 7-21. https://doi.org/10.1038/nrg3606

[7] HUARTE M. The emerging role of lncRNAs in cancer. Nat Med 2015; 21: 1253-1261. https://doi.org/10.1038/nm.3981

[8] QUINN JJ, CHANG HY. Unique features of long non-coding RNA biogenesis and function. Nat Rev Genet 2016; 17 : 47-62. https://doi.org/10.1038/nrg.2015.10

[9] CHEN B, ZHAO Q, GUAN L, LV H, BIE L et al. Long non-coding RNA NNT-AS1 sponges miR-424/E2F1 to promote the tumorigenesis and cell cycle progression of gastric cancer. J Cell Mol Med 2018; 22: 4751-4759. https://doi. org/10.1111/jcmm.13726

[10] CHEN Z, WU J, HUANG W, PENG J, YE J et al. Long NonCoding RNA RP11-789C1.1 Suppresses Epithelial to Mesenchymal Transition in Gastric Cancer Through the RP11789C1.1/MiR-5003/E-Cadherin Axis. Cell Physiol Biochem 2018; 47: 2432-2444. https://doi.org/10.1159/000491617

[11] KONG F, DENG X, KONG X, DU Y, LI L et al. ZFPM2-AS1, a novel $\operatorname{lncRNA}$, attenuates the p53 pathway and promotes gastric carcinogenesis by stabilizing MIF. Oncogene 2018; 37: 5982-5996. https://doi.org/10.1038/s41388-018-0387-9
[12] CHEN P, WANG R, YUE Q, HAO M. Long non-coding RNA TTN-AS1 promotes cell growth and metastasis in cervical cancer via miR-573/E2F3. Biochem Biophys Res Commun 2018; 503: 2956-2962. https://doi.org/10.1016/j. bbrc.2018.08.077

[13] LIN C, ZHANG S, WANG Y, WANG Y, NICE E et al. Functional Role of a Novel Long Noncoding RNA TTN-AS1 in Esophageal Squamous Cell Carcinoma Progression and Metastasis. Clin Cancer Res 2018; 24: 486-498. https://doi. org/10.1158/1078-0432.CCR-17-1851

[14] PEI ZJ, ZHANG ZG, HU AX, YANG F, GAI Y. miR-122$5 p$ inhibits tumor cell proliferation and induces apoptosis by targeting MYC in gastric cancer cells. Pharmazie 2017; 72: 344-347. https://doi.org/10.1691/ph.2017.6404

[15] LIU J, XU J, LI H, SUN C, YU L et al. miR-146b-5p functions as a tumor suppressor by targeting TRAF6 and predicts the prognosis of human gliomas. Oncotarget 2015; 6: 29129_ 29142. https://doi.org/10.18632/oncotarget.4895

[16] ZHANG F, LI K, PAN M, LI W, WU J et al. miR-589 promotes gastric cancer aggressiveness by a LIFR-PI3K/AKT-cJun regulatory feedback loop. J Exp Clin Cancer Res 2018; 37: 152. https://doi.org/10.1186/s13046-018-0821-4

[17] GU J, WANG Y, WANG X, ZHOU D, WANG X et al. Effect of the LncRNA GAS5-MiR-23a-ATG3 Axis in Regulating Autophagy in Patients with Breast Cancer. Cell Physiol Biochem 2018; 48: 194-207. https://doi.org/10.1159/000491718

[18] MO Y, HE L, LAI Z, WAN Z, CHEN Q et al. LINC01287/ miR-298/STAT3 feedback loop regulates growth and the epithelial-to-mesenchymal transition phenotype in hepatocellular carcinoma cells. J Exp Clin Cancer Res 2018; 37: 149. https://doi.org/10.1186/s13046-018-0831-2

[19] ZHANG S, MA H, ZHANG D, XIE S, WANG W et al. LncRNA KCNQ1OT1 regulates proliferation and cisplatin resistance in tongue cancer via miR-211-5p mediated Ezrin/ Fak/Src signaling. Cell Death Dis 2018; 9: 742. https://doi. org/10.1038/s41419-018-0793-5

[20] LI Y, ZENG C, HU J, PAN Y, SHAN Y et al. Long noncoding RNA-SNHG7 acts as a target of miR-34a to increase GALNT7 level and regulate PI3K/Akt/mTOR pathway in colorectal cancer progression. J Hematol Oncol 2018; 11: 89. https://doi.org/10.1186/s13045-018-0632-2

[21] WU R, RUAN J, SUN Y, LIU M, SHA Z et al. Long noncoding RNA HIF1A-AS2 facilitates adipose-derived stem cells (ASCs) osteogenic differentiation through miR-665/ IL6 axis via PI3K/Akt signaling pathway. Stem Cell Res Ther 2018; 9: 348. https://doi.org/10.1186/s13287-0181082-z

[22] LIU H, DENG H, ZHAO Y, LI C, LIANG Y. LncRNA XIST/ miR-34a axis modulates the cell proliferation and tumor growth of thyroid cancer through MET-PI3K-AKT signaling. J Exp Clin Cancer Res 2018; 37: 279. https://doi. org/10.1186/s13046-018-0950-9

[23] NAKAMURA Y, MIGITA T, HOSODA F, OKADA N, GOTOH M et al. Kruppel-like factor 12 plays a significant role in poorly differentiated gastric cancer progression. Int J Cancer 2009; 125: 1859-1867. https://doi.org/10.1002/ ijc. 24538 
[24] MENG YB, HE X, HUANG YF, WU QN, ZHOU YC et al. Long Noncoding RNA CRNDE Promotes Multiple Myeloma Cell Growth by Suppressing miR-451. Oncol Res 2017; 25: 1207-1214. https://doi.org/10.3727/09650401 7X14886679715637

[25] QIN N, TONG GF, SUN LW, XU XL. Long Noncoding RNA MEG3 Suppresses Glioma Cell Proliferation, Migration, and Invasion by Acting as a Competing Endogenous RNA of miR-19a. Oncol Res 2017; 25: 1471-1478. https://doi.org/1 $0.3727 / 096504017 X 14886689179993$

[26] ZHONG X, LU M, WAN J, ZHOU T, QIN B. Long noncoding RNA kcna3 inhibits the progression of colorectal carcinoma through down-regulating YAP1 expression. Biomed Pharmacother 2018; 107: 382-389. https://doi.org/10.1016/j. biopha.2018.07.118

[27] CAO B, LIU C, YANG G. Down-regulation of lncRNA ADAMTS9-AS2 contributes to gastric cancer development via activation of PI3K/Akt pathway. Biomed Pharmacother 2018; 107: 185-193. https://doi.org/10.1016/j.biopha.2018.06.146

[28] WANG P, LI J, ZHAO W, SHANG C, JIANG X et al. A Novel LncRNA-miRNA-mRNA Triple Network Identifies LncRNA RP11-363E7.4 as An Important Regulator of miRNA and Gene Expression in Gastric Cancer. Cell Physiol Biochem 2018; 47: 1025-1041. https://doi.org/10.1159/000490168
[29] ZHAO W, WANG Z, FANG X, LI N, FANG J. Long noncoding RNA Breast cancer antiestrogen resistance 4 is associated with cancer progression and its significant prognostic value. J Cell Physiol 2018. https://doi.org/10.1002/jcp.27962

[30] LI B, SHEN S, ZHANG W, QI T, HU Q et al. Long NonCoding RNA TUG1 as a Potential Novel Biomarker for Predicting the Clinical Outcome of Cancer Patients: a MetaAnalysis. Clin Lab 2018; 64. https://doi.org/10.7754/Clin. Lab.2018.180520

[31] XIONG DD, LI ZY, LIANG L, HE RQ, MA FC et al. The LncRNA NEAT1 Accelerates Lung Adenocarcinoma Deterioration and Binds to Mir-193a-3p as a Competitive Endogenous RNA. Cell Physiol Biochem 2018; 48: 905-918. https://doi. org/10.1159/000491958

[32] CHEN P, FANG X, XIA B, ZHAO Y, LI Q et al. Long noncoding RNA LINC00152 promotes cell proliferation through competitively binding endogenous miR-125b with MCL-1 by regulating mitochondrial apoptosis pathways in ovarian cancer. Cancer Med 2018; 7: 4530-4541. https://doi. org/10.1002/cam4.1547

[33] LIANG H, YU T, HAN Y, JIANG H, WANG C et al. LncRNA PTAR promotes EMT and invasion-metastasis in serous ovarian cancer by competitively binding miR-101-3p to regulate ZEB1 expression. Mol Cancer 2018; 17: 119. https:// doi.org/10.1186/s12943-018-0870-5 